

\title{
Catalytic dehydrogenation of ethylbenzene to styrene over clay-mesoporous silica modified with cobalt oxide com- pound materials
}

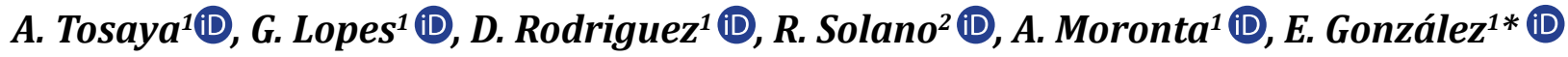

${ }^{1}$ Instituto de Superficies y Catálisis (INSUC). Facultad de Ingeniería. Universidad del Zulia. Maracaibo, 4003, Venezuela.

${ }^{2}$ Grupo Catalizadores y Adsorbentes, Universidad de Antioquia UdeA, Calle 70 No. 52-21, Medellín, Colombia.

*Autor de Contacto: egonzalez@fing.luz.edu.ve

https://doi.org/10.22209/rt.v42n2a05

Recepción: 27/09/2018 | Aceptación: 29/03/2019 | Publicación: 01/05/2019

\begin{abstract}
The catalytic properties of cobalt-modified mesoporous silica-clay compound materials were evaluated for the catalytic dehydrogenation of ethylbenzene to styrene. The solids were prepared with different cobalt oxide contents. Two different methods were used: direct incorporation into the synthesis gel and incipient wetness impregnation. The solids were characterized by X-ray fluorescence, nitrogen adsorption, temperature programmed reduction and X-ray diffraction measurements. The prepared materials showed better textural properties than the starting clay; additionally, they exhibited a type IV isotherm with H3 hysteresis loop. In the impregnated solids, cobalt species were reduced at lower temperature than the ones observed in the materials prepared by incorporation of cobalt into the synthesis gel. The formation of the $\mathrm{Co}_{3} \mathrm{O}_{4}$ phase was evidenced in the materials with higher metal content. The catalytic activity improved with the cobalt content in all of the solids prepared, however, the conversion decreased for the impregnated material with higher cobalt loading, due to the sintering. For the most active catalysts, the results showed a decreased in the selectivity when increasing both the temperature and residence time.
\end{abstract}

Keywords: cobalt oxide, clays, compound materials, ethylbenzene dehydrogenation, mesoporous silica.

\section{Deshidrogenación catalítica de etilbenceno a estireno so- bre materiales compuestos de arcilla-sílice mesoporosa modificada con óxido de cobalto}

\section{Resumen}

Las propiedades catalíticas de materiales compuestos de arcilla-sílice mesoporosa modificada con cobalto fueron evaluadas para la deshidrogenación catalítica de etilbenceno a estireno. Los materiales fueron preparados incorporando diferentes contenidos de óxido de cobalto mediante dos métodos: por incorporación directa dentro del gel de síntesis y por impregnación a humedad incipiente. La caracterización de los sólidos se realizó mediante fluorescencia de rayos X, adsorción física de nitrógeno, reducción a temperatura programa y difracción de rayos X. Los materiales preparados presentaron mejores propiedades texturales que la arcilla de partida, además, mostraron una forma de isoterma de adsorción del tipo IV con lazo de histéresis tipo H3. En los sólidos impregnados las especies de cobalto se redujeron a una temperatura inferior a la observada para los materiales obtenidos por incorporación del cobalto en el gel de síntesis. Se evidenció la formación de la fase $\mathrm{Co}_{3} \mathrm{O}_{4}$ en los materiales con un contenido metálico mayor. La conversión de etilbenceno mejoró con el incremento del contenido de cobalto en ambas series de materiales, sin embargo, la conversión disminuyó para el material impregnado de mayor carga, causado posiblemente por sinterización. La selectividad decreció al aumentar la temperatura y el tiempo de residencia en los ensayos realizados en los catalizadores más activos para cada método de preparación.

Palabras clave: Arcillas, deshidrogenación de etilbenceno, materiales compuestos, óxido de cobalto, sílice mesoporosa. 


\section{Introducción}

La deshidrogenación catalítica de etilbenceno es considerada como una de las reacciones más importantes para la producción de estireno, materia prima para la producción de polímeros y co-polímeros. La deshidrogenación catalítica en presencia de vapor de agua, tiene sus inicios desde la implementación de los primeros procesos industriales en 1930 [1,2]. El mencionado proceso operaba entre $550-700{ }^{\circ} \mathrm{C}$, siendo los catalizadores de óxido de hierro promovidos con óxidos de potasio, cromo o cerio. Sin embargo, a pesar de conducir a altos valores de conversión y selectividad hacia el estireno, el catalizador se desactiva muy rápidamente, debido a: (i) la pérdida de potasio que migra hacia la salida del reactor, (ii) por deposición de coque, (iii) reducción de la fase activa o (iv) por degradación física, haciendo que la vida útil de este tipo de catalizadores sea de 1 a 2 años [3-6].

La necesidad de mejorar los procesos desde el punto de vista económico y ambiental ha promovido el uso de las arcillas, dada su abundancia en la naturaleza y fácil explotación [7]. Diversos materiales han sido desarrollados a partir de arcillas y empleados en una gran variedad de reacciones químicas [8-12]. Además, desde que se extendió el uso surfactantes como orientadores en la síntesis de sólidos mesoporosos, fundamentalmente promovido por la obtención de la familia de sólidos M41S de la Corporación Mobil [13], dos estrategias de síntesis muy similares basadas en el uso de surfactantes permitieron obtener nuevos materiales mesoporos a partir de arcillas naturales o sintéticas, conocidos como: compuestos de arcilla nanoestructurados (CCNs) y arcillas porosas heteroestructuradas (PCHs) [14,15]. Por otra parte, el reto en la ciencia de materiales es implementar métodos de síntesis racionales que permitan la factibilidad de la aplicación industrial de dichos sólidos. En este sentido, Cabrera y col. [16], reportaron un método de síntesis de materiales mesoporosos basado en química suave. La estrategia consistió en la trans-esterificación de un alcóxido metálico con trietanolamina para formar complejos hidrosolubles, conocidos como "complejos atrano". Las principales ventajas son que permite regular la velocidad de hidrólisis de los metales presentes en el gel de síntesis y mantiene el pH en condiciones donde no curre la redisolución de los núcleos de sílice [16,17].

Basados en estos antecedentes, el objetivo de esta investigación fue sintetizar materiales compuestos de arcilla-sílice mesoporosa empleando la ruta de los complejos atranos como método de síntesis. Con la finalidad de proveer propiedades catalíticas a los sólidos, se incorporó cobalto en el gel de síntesis o por el método de impregnación. Los sólidos así obtenidos fueron evaluados en la reacción de deshidrogenación catalítica de etilbenceno a estireno.

\section{Parte Experimental}

\section{Síntesis de los materiales}

Los materiales compuestos se obtuvieron haciendo reaccionar $14,1 \mathrm{~mL}$ de trietanolamina (Fisher Scientific, 99,3\%), (1-x) mol de tetraetilortosilicato (Acros Organic, 98\%) y x mol de $\mathrm{Co}\left(\mathrm{NO}_{3}\right)_{2} \cdot 6 \mathrm{H}_{2} \mathrm{O}$ (Fisher Scientific, 99,8\%), según la relación molar $\mathrm{Si}$ /Co deseada en el gel de síntesis $(5,10,20$ y 40). La mezcla resultante se calentó lentamente hasta $160^{\circ} \mathrm{C}$ bajo agitación durante $30 \mathrm{~min}$. Posteriormente, se bajó la temperatura hasta $120^{\circ} \mathrm{C}$ y se adicionaron $2,7 \mathrm{~g}$ de bromuro de cetiltrimetilamonio (Sigma, 99\%), manteniendo la agitación durante $30 \mathrm{~min}$. Finalmente, se dejó enfriar hasta $80^{\circ} \mathrm{C}$ y se añadieron 50 $\mathrm{mL}$ de una suspensión de arcilla en agua desionizada, manteniendo la agitación por $2 \mathrm{~h}$. La suspensión de arcilla fue preparada previamente mediante la dispersión de la arcilla en agua a $25{ }^{\circ} \mathrm{C}$ y agitación continua por $1 \mathrm{~h}$. Se adicionó la cantidad de arcilla necesaria según la relación arcilla/(Si+Co) de $66 \mathrm{~g} / \mathrm{mol}$. La arcilla utilizada fue una montmorillonita sódica (SWy-2), obtenida del Clay Mineral Repository de la Clay Mineral Society. La arcilla modificada se recuperó por filtración al vacío y se lavó con abundante agua destilada hasta observar la ausencia de espuma en el agua de lavado. Posteriormente, se secó el material a $70^{\circ} \mathrm{C}$ durante $8 \mathrm{~h}$ y se calcinó a $600^{\circ} \mathrm{C}$ por 48 $\mathrm{h}$, empleando una rampa de calentamiento de $2^{\circ} \mathrm{C} / \mathrm{min}$. Los sólidos así sintetizados fueron denominaos: MC-SiCoX para los materiales obtenidos mediante la adición de la sal de cobalto en el gel, donde $\mathrm{X}$ representa la relación molar $\mathrm{Si} /$ Co. Por otra parte se preparó un material siguiendo el mismo procedimiento en ausencia de sales de cobalto, el cual fue denominado MC-Si, que posteriormente fue impregnado con la misma sal de cobalto a diferentes cargas y posteriormente calcinados a la misma temperatura del soporte. Los sólidos así obtenidos fueron denominados; $\mathrm{xCo} / \mathrm{MC}-\mathrm{Si}$, donde " $\mathrm{x}$ " representa el contenido másico de cobalto.

\section{Caracterización}

Se determinó la composición química elemental empleando un espectrómetro de fluorescencia de rayos $\mathrm{X}$, modelo EDX-700HS Shimadzu, bajo el método de polvo en atmósfera de vacío, operando a $50 \mathrm{kV}$ y $30 \mathrm{~mA}$. Las propiedades texturales fueron evaluadas a través de un equipo Micromeritics ASAP 2010. Previo a los análisis, $200 \mathrm{mg}$ de muestras se sometieron un proceso de secado en flujo continuo de argón $(99,99 \%)$ durante $10 \mathrm{~h}$ a una temperatura de $300^{\circ} \mathrm{C}$. Se determinó la reducibilidad de las especies de cobalto mediante ensayos de reducción a temperatura programada, para tal fin, se tomaron 50 mg de muestra, la cual fue previamente sometida a un tratamiento de secado en flujo Ar (99,99\%) de $30 \mathrm{~cm}^{3} /$ min por $1 \mathrm{~h}$ a $120^{\circ} \mathrm{C}$. Posteriormente, se dejó enfriar el reactor hasta temperatura a $25^{\circ} \mathrm{C}$ y se inició la reducción, empleando un flujo de $30 \mathrm{~cm}^{3} /$ min de una mezcla de $\mathrm{H}_{2}$ al $5 \%$ en argón. La temperatura se incrementó en $10^{\circ} \mathrm{C} / \mathrm{min}$ 
hasta $700{ }^{\circ} \mathrm{C}$. La evaluación de la estructura se llevó a cabo en un difractómetro PANalytical Empyrean. Los análisis fueron realizados empleando una radiación $\mathrm{CuK} \alpha$ a $45 \mathrm{kV}$ y $40 \mathrm{~mA}$ bajo una velocidad de barrido correspondiente a $2,4 \theta /$ min con un tamaño de paso de $0,026^{\circ}$, un tiempo por paso de $0,5 \mathrm{~s}$ en el rango de $2-50^{\circ}$ de $2 \theta$.

\section{Actividad catalítica}

Se tomaron $50 \mathrm{mg}$ de catalizador y se trataron en flujo de argón $(99,99 \%)$ de $30 \mathrm{~cm}^{3} /$ min desde $25^{\circ} \mathrm{C}$ hasta la temperaturadereacción $\left(500,600 \mathrm{o} 700^{\circ} \mathrm{C}\right)$ incrementando la temperatura en $10^{\circ} \mathrm{C} / \mathrm{min}$. El etilbenceno se alimentó al reactor por arrastre con una corriente de argón (99,99\%) que burbujea a través de un saturador sumergido en un baño de hielo $\left(\mathrm{P}^{\circ} \mathrm{C}_{10} \mathrm{H}_{8}=0,25 \mathrm{Kpa}, \mathrm{P}^{\circ} \mathrm{Ar}=101,07\right)$. Una vez alcanzada la temperatura de reacción, se hizo pasar el etilbenceno y se procedió a analizar la corriente de salida del reactor mediante cromatografía de gases, empleando un equipo Perkin Elmer Autosystem XL, provisto de un detector de ionización a la llama (FID), y una columna capilar 5\% fenilmetilpolisiloxano.

\section{Resultados y Discusión}

\section{Análisis químico elemental}

La Tabla 1 muestra los resultados del análisis químico elemental obtenido por fluorescencia de rayos $X$. En general, se aprecia el incremento del contenido de silicio, producto de la incorporación de la fase de sílice mesoporosa en la estructura de la arcilla. Adicionalmente, se observa la presencia de cobalto. Las pequeñas cantidades de bromo observadas en los materiales sintetizados evidencia que el surfactante no fue removido completamente durante las etapas de lavado, secado y calcinación, mientras que la disminución de los contenidos de $\mathrm{K}^{+}, \mathrm{Ca}^{2+} \mathrm{y} \mathrm{Mg}^{2+}$ se debe al intercambio de estos cationes por moléculas de surfactante y cationes de cobalto.

\section{Propiedades Texturales}

La modificación de la arcilla con precursores de sílice en presencia de surfactante mejoró las propiedades texturales de manera significativa, contrariamente, la incorporación de cobalto por ambos métodos degradó ligeramente dichas propiedades. La disminución del área específica es atribuida a un bloqueo parcial de los poros luego de la impregnación. Para el caso de los sólidos modificados en síntesis, se observa que incrementan el Vp y Dp, lo que sugiere la formación de poros de mayor tamaño, acompañado de una menor porosidad (Ver Figura 1b).

Las isotermas de adsorción-desorción de nitrógeno mostradas en la Figura 1. Todos los sólidos presentaron una isoterma del tipo IV con un lazo de histéresis del tipo $\mathrm{H} 3$, correspondientes a materiales mesoporosos con poros tipo rendijas no uniformes [18]. El ensanchamiento de lazo de histéresis para el sólido MCSiCo5 es indicativo la contracción de la boca del poro.

La forma de las isotermas fue más definida para el material MC-Si, y no fue afectada por impregnación con cobalto. Solo se observa una disminución en el volumen adsorbido de nitrógeno al incrementar la carga de cobalto. Para la serie de sólidos modificados durante la síntesis se hace menos pronunciado el salto característico del volumen adsorbido de nitrógeno a presiones relativas intermedias, asociado al llenado de los mesoporos. Adicionalmente, el volumen adsorbido de nitrógeno también disminuyó con el aumento de la carga de cobalto.

Tabla 1. Análisis químico elemental y propiedades texturales de los sólidos sintetizados

\begin{tabular}{lcccccccccccc}
\hline \multirow{2}{*}{ Muestra } & $\mathbf{A l}_{2} \mathbf{O}_{3}$ & $\mathbf{B r}$ & $\mathbf{C a O}$ & $\mathbf{C o}_{2} \mathbf{O}_{3}$ & $\mathbf{F e}_{2} \mathbf{O}_{3}$ & $\mathbf{K}_{2} \mathbf{O}$ & $\mathbf{M g O}$ & $\mathbf{M n O}$ & $\mathbf{S i O}_{2}$ & $\begin{array}{c}\mathbf{S}_{\mathbf{B E T}} \\
\mathbf{m}^{2} / \mathbf{g}\end{array}$ & $\begin{array}{c}\mathbf{V}_{\mathbf{p}} \\
\mathbf{~ c m}^{3} / \mathbf{g}\end{array}$ & $\mathbf{D p}_{\text {BJH }}$ \\
\hline SWy-2 & 23,5 & - & 2,9 & - & 8,3 & 1,0 & 2,7 & 0,1 & 61,6 & 28 & 0,07 & 14,4 \\
MC-Si & 13,3 & - & 0,4 & - & 2,7 & 0,4 & 1,5 & - & 81,7 & 680 & 0,57 & 3,1 \\
MC-SiCo5 & 17,4 & 0,1 & 0,9 & 10,4 & 4,5 & 0,4 & 1,9 & - & 64,4 & 409 & 0,65 & 6,0 \\
MC-SiCo10 & 17,5 & 0,1 & 0,7 & 6,9 & 4,5 & 0,4 & 1,9 & - & 68,1 & 496 & 0,79 & 6,0 \\
MC-SiCo20 & 16,5 & - & 0,7 & 2,7 & 4,2 & 0,4 & 2,0 & - & 73,5 & 555 & 0,58 & 4,3 \\
MC-SiCo40 & 17,3 & - & 0,8 & 1,0 & 4,8 & 0,4 & 1,8 & - & 73,8 & 527 & 0,71 & 5,3 \\
\hline 2Co/MC-Si & 13,0 & - & 0,7 & 2,2 & 3,2 & 0,3 & - & - & 80,5 & 622 & 0,65 & 4,2 \\
4Co/MC-Si & 12,7 & - & 0,7 & 3,5 & 2,9 & 0,3 & 1,3 & - & 78,6 & 619 & 0,68 & 4,4 \\
10Co/MC-Si & 12,2 & 0 & 0,6 & 9,5 & 2,9 & 0,3 & 1,9 & - & 72,6 & 579 & 0,63 & 4,4 \\
\hline
\end{tabular}



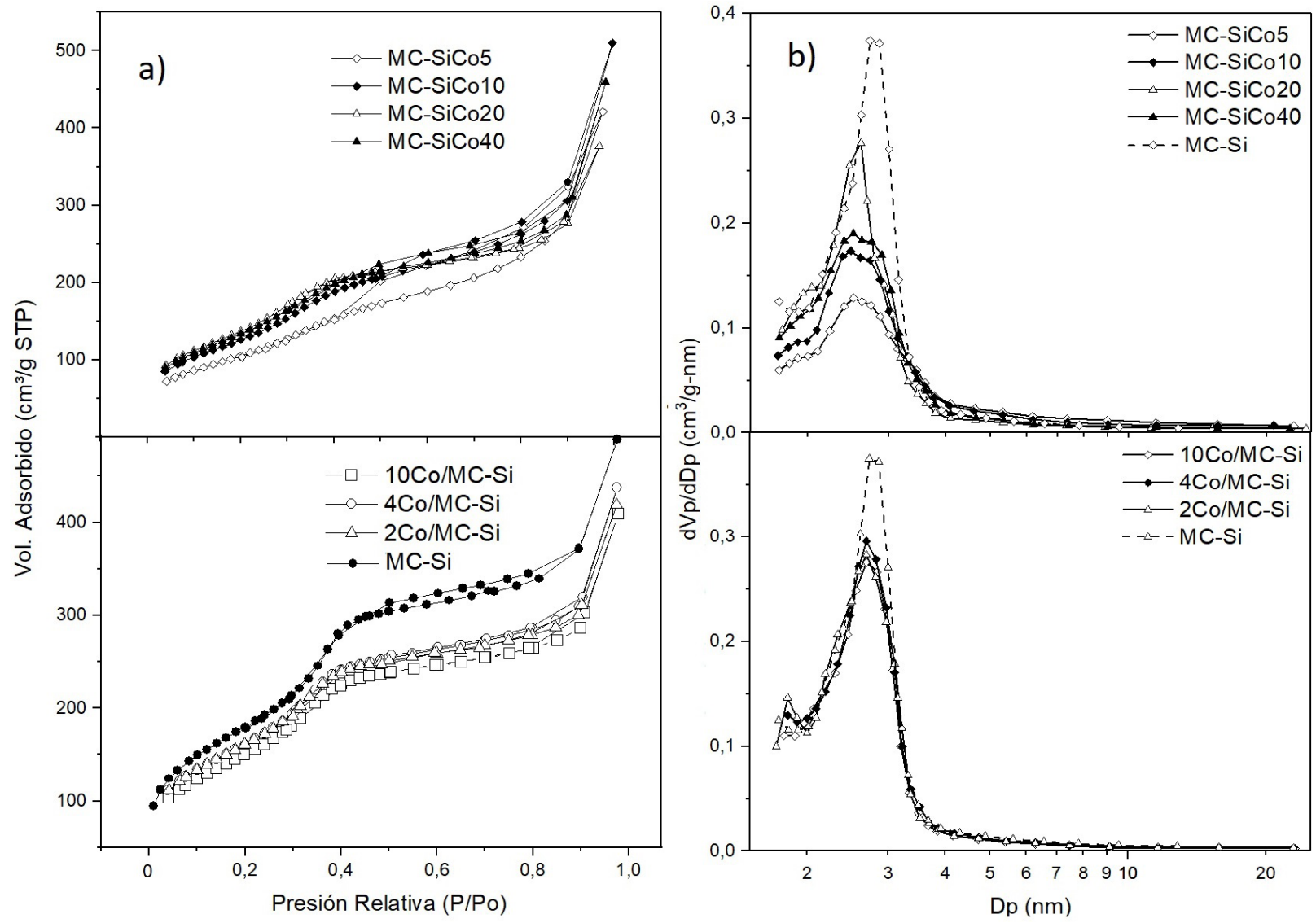

Figura 1. a) Isotermas de adsorción-desorción de nitrógeno y b) Curvas de distribución de tamaño de poro de los sólidos sintetizados.

La distribución de tamaños de poro de los materiales modificados son mostradas en la Figura $1 \mathrm{~b}$. Las curvas muestran una distribución estrecha y centrada alrededor de los $3 \mathrm{~nm}$, similar al reportado por Qing y col. [19]. La incorporación de cobalto por ambos métodos disminuyó la porosidad y ensanchó ligeramente la curva de distribución.

\section{Naturaleza de la fase de óxido de cobalto}

Los perfiles de reducción de los materiales modificados son mostrados en la Figura 2. Cuando el cobalto se incorporó en el gel de síntesis se observó una señal de reducción entre 560 y $623{ }^{\circ} \mathrm{C}$ y un pequeño hombro entre $438-554^{\circ} \mathrm{C}$, además de un ensanchamiento progresivo a medida que aumenta el contenido de cobalto. Respecto a los materiales obtenidos por impregnación, estos mostraron señales de reducción a temperaturas inferiores. Las señales observadas entre 200 y $400{ }^{\circ} \mathrm{C}$ son atribuidas a la reducción de especies $\mathrm{Co}_{3} \mathrm{O}_{4}$ a $\mathrm{CoO}$, mientras que las señales observadas a temperaturas mayores a los $550{ }^{\circ} \mathrm{C}$ son asociadas a la reducción de $\mathrm{CoO}$ a $\mathrm{CoO}[20,21]$.
${ }_{\mathrm{L}}$ os cambios en el perfil de reducción del cobalto con el método de síntesis, indican que la incorporación de los precursores del óxido de cobalto en el gel de síntesis, conduce a la formación de especies con una mayor interacción con la estructura, dado que la reducción de las especies de $\mathrm{Co}^{3+}$ ocurre a una mayor temperatura. Los desplazamientos en las señales de reducción pueden ser atribuidos a que en el método de impregnación se genera un $\mathrm{pH}$ ácido, que hace que el metal simplemente se deposite sobre el soporte y no presente interacciones metal-soporte considerables, mientras que el pH básico de la vía de los complejos atranos favorece la formación de especies superficiales de cobalto tipo filosilicatos.

En la Figura 3 se muestran los difractogramas de rayos $\mathrm{X}$ de los materiales sintetizados. En ella se puede apreciar que los sólidos con mayor carga mostraron señales de difracción muy tenues, correspondiente a la fase cúbica de $\mathrm{Co}_{3} \mathrm{O}_{4}$, lo cual es consistente con los perfiles de reducción obtenidos. La ausencia de picos o la baja intensidad de los mismos, sugiere que predomina 


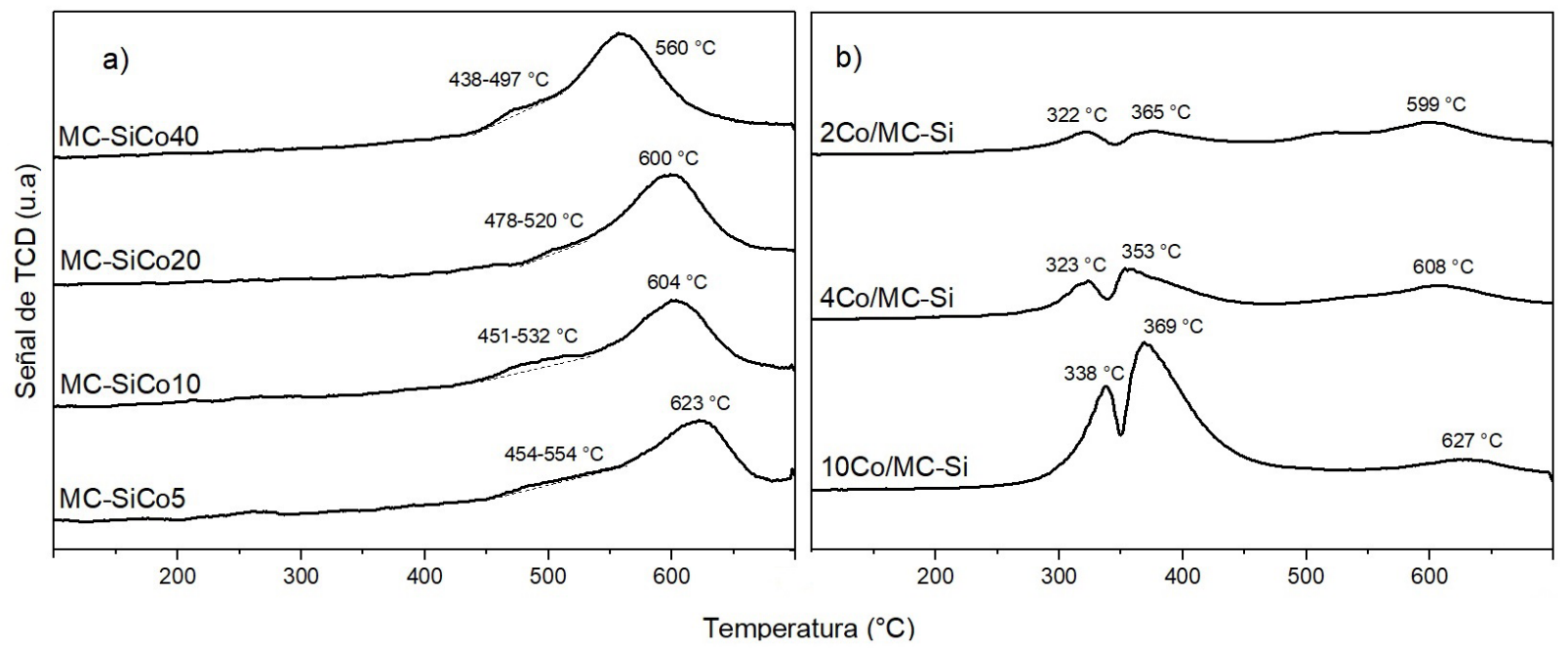

Figura 2. Perfiles de reducción a temperatura programada de los sólidos sintetizados.

la formación de fases amorfas o que la fase cristalina está constituida por pequeños cristales, posiblemente muy dispersos sobre la superficie del catalizador [21]

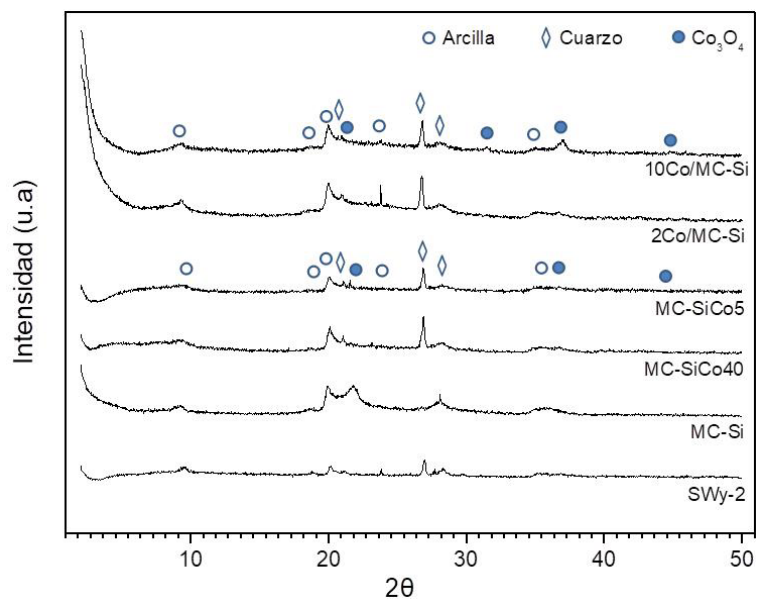

Figura 3. Perfiles de XRD de los sólidos sintetizados.

\section{Actividad catalítica}

Los resultados de la actividad catalítica se muestran en la Figura 4. Todos los materiales mostraron una conversión baja, la cual fue constante luego de $15 \mathrm{~min}$ de reacción. Esta observación es típicamente debida a las restricciones termodinámicas, por lo que industrialmente se utiliza vapor de agua para favorecer la conversión [22]. Los materiales sintetizados mediante la incorporación de cobalto en el gel de síntesis mostraron una conversón inicial ligeramente mayor a la conversión en estado estacionario. La ausencia de benceno es debida a la baja acidez del soporte, o a una rápida desactivación de los sitios ácidos formación de coque.

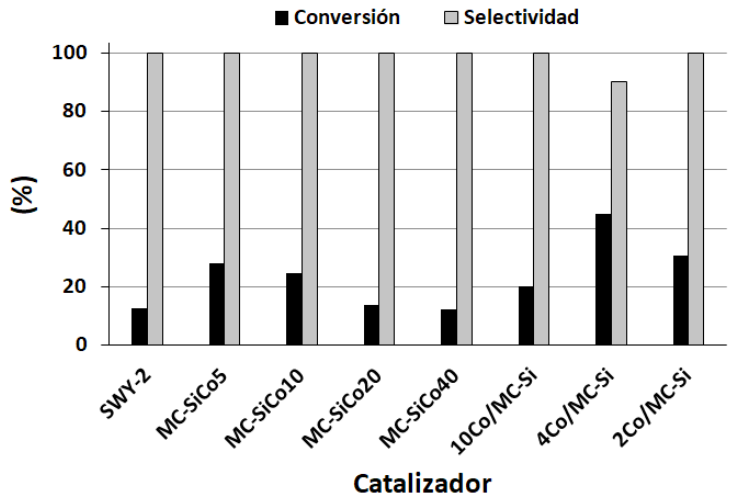

Figura 4. Valores de conversión y selectividad hacia estireno para la deshidrogenación de etilbenceno a $600^{\circ} \mathrm{C}$ luego de $2 \mathrm{~h}$ de reacción.

Contrariamnete, una alta selectividad a benceno en los primeros minutos de reacción es observada típicamente en catalizadores de cromo $[2,23]$. En nuestro caso, la elevada selectividad hacia estireno desde los primeros minutos de reacción pudiese ser atribuida a la desactivación de los sitios ácidos superficiales responsables del craqueo u oligomerización durante la etapa remoción del surfactante mediante calcinación.

Los resultados también indican que la arcilla no modificada fue activa hacia la deshidrogenación de etilbenceno, con una conversión alrededor del 13\%. Dicha actividad es atribuida a la presencia de hierro $(8,3 \% \mathrm{~m} / \mathrm{m})$ en las láminas de la arcilla, dado que es bien conocido que éstas especies son muy activas para ésta reacción [24]. Para la serie de compuestos donde el cobalto se adicionó directamente en el gel, la actividad aumentó de forma proporcional al contenido metálico, mientras que los materiales obtenidos por impregnación evidenciaron un comportamiento similar, pero con la 
máxima carga metálica ocurrió una pérdida drástica de la actividad catalítica, causada posiblemente por la disminución de la superficie metálica por sinterización. Por otra parte, la muestra 4Co/MC-Si a pesar de ser la más activa, también fue la que mostró una menor selectividad, comportamiento que no fue observado para la serie modificada en síntesis.

A pesar que los materiales MC-SiCo5 y 10Co/ MC-Si tienen un contenido metálico similar, el sólido preparado por impregnación fue menos activo respecto al material obtenido mediante la incorporación del cobalto en el gel de síntesis. Al comparar dicho comportamiento con los otros materiales obtenidos por impregnación, se observa que también mostró una actividad menor, por lo que la formación de partículas de mayor tamaño y consecuente disminución de la dispersión de la fase activa, puede ser la causa principal de la pérdida de sitios activos. En ambas series de materiales se aprecia la dependencia de la actividad catalítica con el contenido de cobalto, adicionalmente, el método de síntesis afectó el desempeño catalítico, lo que sugiere que la menor actividad de los sólidos preparados mediante la incorporación del cobalto en el gel de síntesis es causada posiblemente por la formación de especies tipo silicatos, ya que dichas especies, también se redujeron a mayor temperatura.

En las Figura 5 muestra el efecto de la temperatura y de la velocidad espacial sobre la selectividad y desempeño catalítico de los sólidos que mostraron la mayor actividad en ambas series de materiales. La conversión fue directamente proporcional a la temperatura de reacción, comportamiento que es acorde a la naturaleza endotérmica de la reacción.
El material 4Co/MC-Si impregnado mostró mayor actividad y menor selectividad que el material MCSiCo5 a temperaturas mayores de $500^{\circ} \mathrm{C}$. El incremento de la temperatura de reacción favoreció la formación de benceno y tolueno como productos secundarios de las reacciones de craqueo e hidrogenólisis, siendo la pérdida de selectividad más notable para el catalizador impregnado, donde los productos secundarios se observaron a $600{ }^{\circ} \mathrm{C}$, mientras que el catalizador impregnado mostró $100 \%$ de selectividad a la misma temperatura.

Respecto a la velocidad espacial, la conversión fue inversamente proporcional a ésta para ambos tipos de materiales, comportamiento esperado, ya que una menor velocidad espacial implica un mayor tiempo de residencia y/o contacto. El catalizador preparado por síntesis mostró ser más selectivo hacia la formación de estireno que el catalizador impregnado. Para el caso de $0,7 \mathrm{~h}^{-1}$ se ve favorecida la reacción secundaria de craqueo (benceno) seguida de la reacción de hidrogenólisis (tolueno). Para $0,4 \mathrm{~h}^{-1}$ la reacción secundaria más favorecida sigue siendo el craqueo para producir benceno, mientras que para $0,2 \mathrm{~h}^{-1}$ los productos de oligomerización fueron los mayoritarios. Esta tendencia indica que a menor velocidad espacial predomina la producción de precursores de coque por oligomerización del estireno [2]. La distribución y productos de reacción observados en este trabajo son similares a los reportados por Gómez y col [2] para catalizadores de cromo soportado sobre alúmina, siendo el benceno y tolueno los productos principales observados en los primeros instantes de la reacción, y que luego de la
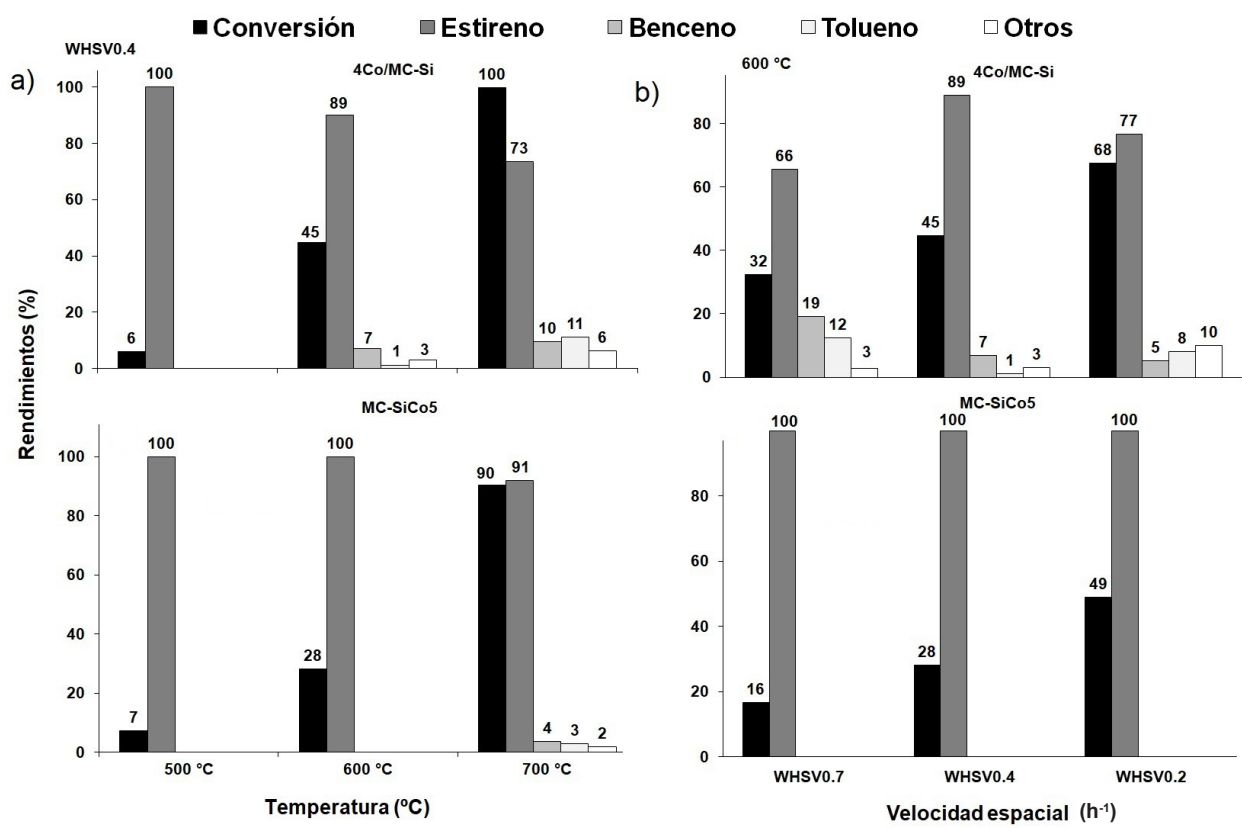

Figura 5. Efecto de la temperatura (a) y de la velocidad espacial (b) sobre la conversión y la distribución de productos de reacción para los materiales $4 \mathrm{Co} / \mathrm{MC}-\mathrm{Si}$ y MC-SiCo5. $\mathrm{P}^{\circ} \mathrm{C} 10 \mathrm{H} 8=0,25 \mathrm{Kpa}, \mathrm{P}^{\circ} \mathrm{Ar}=101,07$. 
rápida desactivación de los sitios responsables de craqueo e hidrogenólisis, incrementa la selectividad a estireno y se alcanza rápidamente el estado estacionario. Todo esto confirma que la deshidrogenación catalítica es un proceso complejo donde ocurren varias reacciones simultáneas, por lo que las condiciones de reacción y la naturaleza de las especies catalíticamente activas son determinantes en el desempeño catalítico y la selectividad.

\section{Conclusiones}

Se sintetizaron materiales mesoporosos con cobalto a partir de arcillas, con propiedades catalíticas para la deshidrogenación de etilbenceno a estireno. El incremento del contenido de cobalto disminuye las propiedades texturales de los materiales, dado que involucra la contracción del tamaño de poro o bloqueo de los mismos. Los difractogramas de rayos-X reflejaron únicamente la presencia de especies de cobalto en su forma más estable $\left(\mathrm{Co}_{3} \mathrm{O}_{4}\right)$, las cuales se encuentran altamente dispersas dada la baja intensidad de la señal. La actividad fue dependiente del método de síntesis, siendo los sólidos impregnados los más activos. La incorporación del cobalto a través del gel de síntesis conduce posiblemente a especies tipo silicatos que son menos activas, los cuales además son difíciles de reducir según los ensayos de reducción a temperatura programada. La deshidrogenación catalítica de etilbenceno se favoreció con el aumento de la temperatura y menor velocidad espacial, sin embargo, la selectividad decreció, ya que un aumento de la temperatura o tiempo de residencia favorece las reacciones colaterales de craqueo u oligomerización.

\section{Agradecimientos}

Los autores agradecen al Grupo de Investigación en Catalizadores y Adsorbentes de la Universidad de Antioquia, Colombia y al Consejo de Desarrollo Técnico, Científico y Humanístico de la Universidad del Zulia (CONDES-LUZ) por el financiamiento otorgado a la presente investigación mediante el Proyecto CC-191-17.

\section{Referencias Bibliográficas}

[1] Irún O., Sadosche S., Lasobras J., Soler J, Francés E., Herguido J., Menéndez M.: "Catalysts for the production of styrene from ethylbenzene: Redox and deactivation study". Catal. Today 203 (2013) 5359.

[2] Gomez S., McMillan L., McGregor J., Axel J., Al-Yassir N., Al-Khattaf S., Gladden L.: "New perspective on catalytic dehydrogenation of ethylbenzene: the influence of side-reactions on catalytic performance". Catal. Sci. Technol., Vol. 5 (2015) 3782-3797.
[3] Mediero M., McGregor J., McMillanL., Al-Yassir N., Bingham P., Forder S., Gorin C., Al-Khattaf S., Gladden L., Midgley P.: "Structural changes in $\mathrm{FeOx} / \gamma-\mathrm{Al}_{2} \mathrm{O}_{3}$ catalysts during ethylbenzene dehydrogenation". Catal. Struct. Reactivity, Vol. 2, No. 1 (2016) 25-32

[4] Cavani F. Trifiro F.: "Alternative processes for the production of styrene”. Appl. Catal A., 133 (1995) 219-239.

[5] Mihajlova A., Andreev A., Shopov D., Dimitrova R.: "Effect of the precursor properties on the selectivity of iron based catalysts in the dehydrogenation of ethylbenzene to styrene". Appl. Catal. A., Vol. 40, No.1, (1988) 247-253.

[6] Rossetti I., Bencini E., Trentini L., Forni L.: "Study of the deactivation of a commercial catalysts for ethylbenzene dehydrogenation to styrene". Appl. Catal. A, Vol. 292, No.1 (2005) 118-123.

[7] Zhou C.: "An overview on strategies towards claybased designer catalysts for green and sustainable catalysis". Appl. Clay Sci., Vol. 53, No. 1 (2011) 87-96.

[8] Mccabe R., Adans J.: "Clay Minerals as Catalysts". Developments in Clay Science, Handbook of Clay Science, Vol. 5 (2013) 491-538.

[9] DeStefanis, A., Tomlinson A.A.G.: "Towards designing pillared clays for catalysis". Catal. Today, Vol. 114, No. 1 (2006) 126-141.

[10] Betega L., Morales A., Valenzuela F.: “Organoclays: properties, preparation and applications". Appl Clay Sci, Vol. 42, No.1, (2008) 8-24.

[11] Pinto L., Saini V., Guil J., Pires J.: "Introduction of aluminum to porous clay heterostructures to modify the adsorption properties". Appl. Clay Sci., Vol. 101, No.1 (2014) 497-502.

[12] Zhou C., Keeling J.: "Fundamental and applied research on clay minerals: From climate and environment to nanotechnology". Appl. Clay Sci., Vol. 74, No. 1 (2013) 3-9.

[13] Beck J. Vartuli J., Roth W., Leonowicz M, Kresge C., Schmitt D., Chu C., Olson D., Sheppard E., McCullen S., Higgins J., Schlenkert J.: "A new family of mesoporous molecular sieves prepared with liquid crystal templates". J. Am. Chem. Soc., Vol. 114, No.27 (1992) 10834-10843.

[14] Zhou H., Lu G.: "Engineering the structures of 
nanoporous clays with micelles of alkyl polyether surfactants". Langmuir, Vol. 17, No. 3 (2001) 588594.

[15] Polverejan M., Liu Y., Pinnavaia T.: "Aluminated derivatives of porous clay heterostructures (PCH) assembled from synthetic saponite clay: Properties as supermicroporous to small mesoporous acid catalysts". Chem. Mater., Vol. 14, No. 5 (2002) 22832288.

[16] Cabrera S. El Haskouri J., Guillen C., Latorre J., Beltrám A., Beltrán D., Marcos M., Amorós P.: "Generalised syntheses of ordered mesoporous oxides: The atrane route". Solid State Sci., Vol. 2, No. 1 (2000) 405-420.

[17] Huerta L., Guillen C., Latorre J., Beltrán A., Martínez R., Marcos M., Beltrán D., Amorós P.: "Bases for the synthesis of nanoparticulated silicas with bimodal hierarchical porosity". Solid State Sci., Vol. 8, No.1 (2006) 940-951.

[18] Thommes M, Kaneko K., Neimark A., Olivier J., Rodriguez F., Rouquerol J., Sing K.: "Physisorption of gases, with special reference to the evaluation of surface area and pore size distribution (IUPAC Technical Report)". Pure Appl. Chem., Vol 87, No. 9-10 (2015) 1051-1069.

[19] Qing H, Zhong L., Bingsen Z., Guang W., Chunyan M., Wiebke F., Jinjun L., Zhao L., Zhengping H., Dang S.: "Porous montmorillonite heterostructures directed by a single alkyl ammonium template for controlling the product distribution of Fischer-Tropsch synthesis over Cobalt". Chem. Mater., Vol 24, No. 6 (2012) 972-974.
[20] Zhao Y. H., Haou Q. Q., Song Y.H., Fan W. B., Liu Z.W.: "Cobalt Supported on Alkaline-Activated Montmorillonite as an Efficient Catalyst for FischerTropsch Synthesis". Energy Fuels, Vol. 27, No.11 (2013) 6362-6371.

[21] Chromcáková Z., Obalová L., Kovanda F., Leguta D., Titov A., Ritz M., Fridrichová D., Michalika S, Kustrowski P., Jirátová K.: "Effect of precursor synthesis on catalytic activity of $\mathrm{Co}_{3} \mathrm{O}_{4}$ in $\mathrm{N}_{2} \mathrm{O}$ decomposition". Catal. Today, Vol. 257, No. 1 (2015) 18-25.

[22] Ranke W.: "Nanostructured Catalysts: Selective Oxidations”. Royal Soc. Chem., London, 2011.

[23] Vicente M., Meyer A., González E., Bañares A., Gandía L., Gil A.: "Dehydrogenation of ethylbenzene on alumina-chromia-pillared saponites". Catal. Letters, Vol. 78 (2002) 1-4.

[24] Ferreira N., Filho J., Oliveira A.: "Porous ternary Febased catalysts for the oxidative dehydrogenation of ethylbenzene in the presence (absence) of carbon dioxide". RSC Adv., Vol. 5, No. 27 (2015) 2090020913. 


\section{REVISTA TECNICA}

DE LA FACULTAD DE INGENIERIA

UNIVERSIDAD DEL ZULIA

Vol. 42. N², Mayo-Agosto 2019, pp. 47 - 97

Esta revista fue editada en formato digital y publicada en Abril de 2019, por el Fondo Editorial Serbiluz, Universidad del Zulia. Maracaibo-Venezuela

www.luz.edu.ve

www.serbi.luz.edu.ve

produccioncientifica.luz.edu.ve 